\title{
Structural and Optical Characterization of Thiourea-Poly (Vinyl Alcohol) Composites
}

\author{
T. Fahmy ${ }^{* 1,2}$, A. $\operatorname{sarhan}^{2}$ and Z. M. Elqahtani ${ }^{3}$ \\ ${ }^{l}$ Dept. of Physics, College of Science and Humanities in Al-Kharj, Prince Sattam bin Abdulaziz University, Al-Kharj 11942, Saudi Arabia. \\ ${ }^{2}$ Polymer Research Group, Dept. of Physics, Faculty of Science, Mansoura University, 35516 Mansoura, Egypt. \\ ${ }^{3}$ Dept. of Physics, College of Science, Princes Nourah bint Abdulrahman University, Ryiadh, Saudi Arabia.
}

https://orcid.org/0000-0002-5078-2245

${ }^{*}$ Corresponding author

\begin{abstract}
The present manuscript reports the effect of thiourea doping on the optical properties of poly (vinyl alcohol), PVA. Films of thiourea doped PVA samples have been prepared using casting method. Different techniques such as, XRD, FTIR, Raman spectroscopy and UV-Vis spectroscopy have been used to investigate the prepared samples. XRD results show that thiourea is highly crystalline material with an intense sharp peak at $2 \theta=20.69^{\circ}$ and the crystallinity of thiourea doped PVA samples increased with increasing the thiourea content. FTIR and Raman spectroscopy are used to investigate the change in the molecular structure PVA-thiourea composites. The optical constants of thiourea doped PVA samples have been estimated using the analysis of UV-Vis data. It is found that, values of absorption edge, direct and indirect optical gap energy are decreased with doping thiourea salt until a concentration of $5 \mathrm{wt} \%$ and then start to increase again. Values of dispersion energy $\left(E_{d}\right)$, single oscillator energy $\left(E_{o}\right)$ and different parameters of dielectric constant have been estimated.
\end{abstract}

Keywords: PVA, Thiourea, Optical gap, dispersion energy, single oscillator energy, Lattice dielectric constant.

\section{INTRODUCTION}

Polyvinyl alcohol, (PVA) is characterized by carbon chain backbone linked with hydroxyl groups. The hydroxyl groups, $\mathrm{OH}$ groups, are considered as a source of hydrogen bonding, therefore, it helps to form the polymer blends or polymer complexes [1-4]. PVA is water-soluble and biocompatible polymer and is vastly used in the preparation of polymer blends due to its excellent physical and chemical properties [5]. So the applications of PVA can be increased more and more by adding different dopant materials into PVA matrix [6].

Thiourea is considered as an interesting inorganic modifier matrix because of its higher polarity and its ability to compose an extensive hydrogen bonds network [7]. Thiourea crystals also show the piezoelectric effect and it is an organic nonlinear optical material and can be used in many applications, such as, frequency conversion, harmonic generators, dynamic holography, frequency mixing, light modulation and optical memory storage $[8,9]$.

The optical and electrical properties of polymer blends and polymer composites have been studied intensively [10-15]. The investigation of optical absorption spectrum of solids gives more details about both optical energy gap as well as the band structure of amorphous and crystalline materials. Information about atomic vibration and electronic states can be obtained by the analysis of absorption spectrum in the lower energy and higher energy regions, respectively [16-19]. In this manuscript, the effect of thiourea dopant on the structure and optical properties of PVA has been investigated.

\section{EXPERIMENTAL WORK}

\section{Materials}

Poly (vinyl alcohol) (PVA) is a commercial product, purchased from Merck, (Germany), with molecular weight equals to 72000 and thiourea salt after recrystallization, $\mathrm{M}_{\mathrm{w}}$ 76.12, are supplied by BDH Chemicals Ltd, Poole, (England) and El-Nasr Co., (Egypt), respectively.

\section{Sample Preparation}

Doped PVA samples with different ratio of thiourea are prepared using casting method. PVA and thiourea are dissolved in distilled water for few hours at $\mathrm{T}=343 \mathrm{~K}$. The solution is continuously stirred for two hours to get a homogeneous mixture. The solution is then casted in petri dish in an oven regulated at $\mathrm{T}=343 \mathrm{~K}$ for few days.

$\mathrm{X}$-ray diffraction pattern of all samples is carried out using Rigaku Ultima IV diffractometer with $\mathrm{Cu}-\mathrm{K} \alpha$ radiation, $(\lambda=$ $1.5418 \AA$ at $40 \mathrm{kV}$ ), (at Central Lab, College of Science, Prince Sattam bin Abdulaziz Uni, KSA). FT-IR spectra are carried out between 400 and $4000 \mathrm{~cm}^{-1}$ by FT-IR spectroscopy, Mattson, UK (at Chemistry Dept., Faculty of Sci., Mansoura Uni. Egypt). Raman Spectroscopy is performed in the range $3650-50 \mathrm{~cm}^{-1}$ using Senterra II, Bruker (at Central Lab, College of Science, Prince Sattam bin Abdulaziz Uni, KSA). UV-Vis spectra are recorded between 200 and $800 \mathrm{~nm}$ using UV/Vis Unicom, Mattson, UK (at Chemistry Dept., Faculty of Sci., Mansoura Uni. Egypt). 


\section{RESULTS AND DISCUSSION}

\section{XRD}

X-ray diffraction study of polymer and polymer composite is useful technique to investigate the amorphous and crystalline nature of the materials. Fig. 1 represents XRD spectrum of thiourea and thiourea doped PVA samples. The reflection peaks of thiourea are detected at 20, 20.69, 23.29, 28.51, 29, $31.41,5.53,42.33,47.16,48.86$ and $51.66^{\circ}$, as shown in Fig. 1a. These reflections are consistent with characteristic peaks of the typical thiourea salt [20]. Fig. 1b displays XRD spectra of PVA and doped PVA samples with different concentrations of thiourea salt. It is found that XRD of pure PVA is

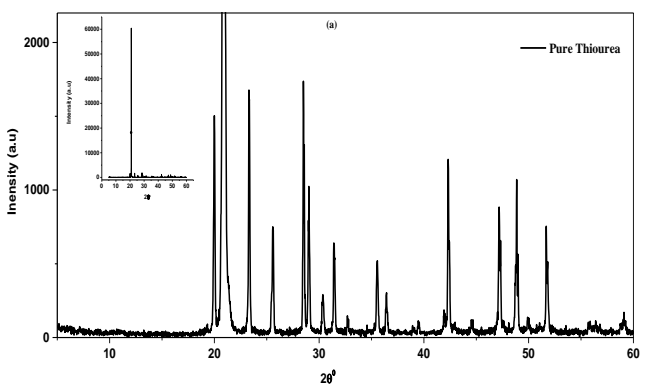

Fig. 1a: XRD spectrum of pure Thiourea.

\section{FTIR-Spectroscopy}

FTIR spectroscopy is useful to define the functional groups and to investigate the interactions and complexation in the polymeric materials. Fig. 2 displays FTIR spectra of PVA and thiourea doped PVA samples in the wavenumber region from 4000 to $400 \mathrm{~cm}^{-1}$. It is observed that, FTIR spectrum of PVA, Fig. 2a, is characterized by many absorption bands, such as, the absorption bands in the range of $3400-3235 \mathrm{~cm}^{-1}$ are assigned to $\mathrm{OH}$ intermolecular hydrogen bonded. The absorption bands at $1720 \mathrm{~cm}^{-1}, 1564 \mathrm{~cm}^{-1}$ and $1427 \mathrm{~cm}^{-1}$ are attributed to and $\mathrm{C}=\mathrm{O}$ stretching, $\mathrm{C}-\mathrm{H}$ bending of $\mathrm{CH}_{2}$ and COO- stretching vibrations, respectively. The bands at 1333 , 1250 and $1092 \mathrm{~cm}^{-1}$ are due to the interaction between $\mathrm{O}-\mathrm{H}$ bending and C-O stretching. The bands at 916 and $848 \mathrm{~cm}^{-1}$ provide more information on the ratio of syndiotactic and characterized by a semicrystalline peak at $2 \theta=19.89^{\circ}$, as reported elsewhere, [21]. The intensity and position of this peak is changed with doping, indicating that the crystallinity of doped PVA samples has been enhanced. One can see that, highly doped PVA sample is characterized by an amorphous halo at $2 \theta=9.15^{\circ}$ and many reflection peaks at $2 \theta=20.18$, $20.98,23.42,25.63,28.58,29.11,31.43,35.66$ and $36.41^{\circ}$. The higher degree of crystallinity depends mainly on the regular arrangement of molecules of the materials. Such behavior has been reported previously, thiourea may enhance effective delocalization of PVA chains leading to the ordering of polymer chains of PVA along the thiourea chains [22,23].

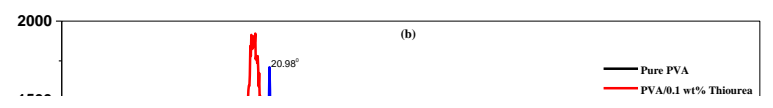

Fig. 1b: XRD spectra of PVA and doped PVA with varying thiourea concentrations.

isotactic sequences in PVA matrix [24].

On the other hand, Fig. 2e shows FTIR of pure thiourea with characteristic absorption bands. The bands at $3378 \mathrm{~cm}^{-1}, 3269$ $\mathrm{cm}^{-1}$ and $3172 \mathrm{~cm}^{-1}$ are attributed to different types of asymmetric and symmetric stretching $\mathrm{NH}_{2}$ groups. Absorption bands at $2667 \mathrm{~cm}^{-1}, 2353 \mathrm{~cm}^{-1}$ and $2104 \mathrm{~cm}^{-1}$ are attributed to ammonium and iminium salt like structure. The band at $1617 \mathrm{~cm}^{-1}$ is attributed to thioamide $(\mathrm{C}=\mathrm{S}$ linked to nitrogen), asymmetric stretching vibration, whereas, the band at $1469 \mathrm{~cm}^{-1}$ is assigned to antisymmetric (NCN) stretching of the thiourea [25]. The absorption bands at $1087 \mathrm{~cm}^{-1}, 729 \mathrm{~cm}^{-}$ ${ }^{1}, 632 \mathrm{~cm}^{-1}$ and $492 \mathrm{~cm}^{-1}$ are assigned to $\mathrm{C}-\mathrm{N}$ symmetric stretching vibrations, $\mathrm{C}=\mathrm{S}$ symmetric stretching vibrations, $\mathrm{C}$ $\mathrm{N}$ bending vibration and $\mathrm{N}=\mathrm{C}=\mathrm{N}$ rocking mode, respectively [26].

Fig. 2: FTIR spectrum of (a) pure PVA, (b-d) doped samples and (e) pure Thiourea. 
Figs. (2b-d) display FTIR of thiourea doped PVA samples to investigate the local bonding structure in PVA. These bonds are affected after doping the polymer structure of PVA with thiourea salt. The strong hydrogen bonding between both PVA and thiourea salt leads to the absorption bands to become sharper and shifted to higher frequencies in the range of $3300-3500 \mathrm{~cm}^{-1}$. The band at $1720 \mathrm{~cm}^{-1}(\mathrm{C}=\mathrm{O})$ of PVA has been shifted to lower wavenumber $\sim 1702 \mathrm{~cm}^{-1}$. The absorption band at $1092 \mathrm{~cm}^{-1}$ which is measured the crystallinity degree of pure PVA is shifted to higher wavenumber at $1127 \mathrm{~cm}^{-1}$. The shift in the band positions to higher frequency values can be ascribed to greater double bond character of $\mathrm{C}=\mathrm{N}$ during the formation of PVA-thiourea complex [20]. Moreover, some of absorption bands having different intensities and positions are detected which are attributed to different bending and stretching vibrations, such as, $2937 \mathrm{~cm}^{-1}, 1564 \mathrm{~cm}^{-1}, 1333 \mathrm{~cm}^{-1}$ and $848 \mathrm{~cm}^{-1}$, respectively

\section{Raman Spectroscopy}

Raman spectroscopy is a non-destructive technique to characterize the crystalline materials. It is intensively used to define functional groups for knowing the molecular structure of polymer complexes and composites and it can be used also in conformational analysis of samples. In general, chemical bonds containing heavy atoms show an intense Raman band compared to those containing lighter atoms. Hence, the Raman scattering intensity produced by the double bond vibration is much stronger than that produced by single bond vibration [27]. Fig. 3 displays Raman spectrum of PVA/0.5 $\mathrm{wt} \%$ thiourea, as a representative sample of doped samples in the $50-3500 \mathrm{~cm}^{-1}$ region.

Fig. 3: Raman spectrum of PVA/0.5 wt $\%$ thiourea.

The main characteristic Raman bands of thiourea have been observed at 1371, 1094, 733 and $486 \mathrm{~cm}^{-1}$. These bands are assigned to N-C-N symmetric stretching modes, strong coupling of $\mathrm{C}-\mathrm{N}$ stretching with the $\mathrm{NH}_{2}$ group rocking, C-S stretching and $\mathrm{N}-\mathrm{C}-\mathrm{N}$ bending, respectively. On the other hand, because of the low polarizability and low intensity, SCNN out-of-plane bending mode can't be observed in this present Raman spectrum, but it is found at $\sim 632 \mathrm{~cm}^{-1}$ in FTIR spectrum of thiourea, as shown in Fig. 2e [28]. A broad band has been observed at $3278 \mathrm{~cm}^{-1}$ and is raised from $\mathrm{OH}$ stretching, indicating that the presence of hydroxyl groups. The band with a maximum at $2912 \mathrm{~cm}^{-1}$ is related to the vibrations of valence $\mathrm{C}-\mathrm{H}$, whereas, the band at $1371 \mathrm{~cm}^{-1}$ is attributed to the vibrations of valence $\mathrm{C}-\mathrm{O}$ of secondary alcohol. The region $1200-1000 \mathrm{~cm}^{-1}$ consists of different numbers of modes are sensitive to the crystallinity degree in PVA [29]. The bands at $924 \mathrm{~cm}^{-1}, 856 \mathrm{~cm}^{-1}$ and $418 \mathrm{~cm}^{-1}$ are attributed to twist mode of syndiotactic, isotactic sequences and $\mathrm{C}-\mathrm{H}$ out of plane vibration, respectively [30].

\section{UV-Vis Spectra}

UV-Vis spectroscopy of PVA/thiourea composite samples have been investigated between 200 and $800 \mathrm{~nm}$. PVA spectrum is characterized by three bands at 278, 313 and 410 $\mathrm{nm}$, as observed in Fig. 4. The band at $278 \mathrm{~nm}$ is related to the absorption of carbonyl group $(\mathrm{C}=\mathrm{O})$, while, band at $313 \mathrm{~nm}$ is related to $-\mathrm{CO}-(\mathrm{C}=\mathrm{C})_{3}$-group $[31,4]$. UV spectrum of pure thiourea salt has been characterized by three bands at 237,270 and $350 \mathrm{~nm}$, respectively. The band at $270 \mathrm{~nm}$ which is observed as a shoulder is attributed to $n \rightarrow \pi^{*}$ transition, whereas, the band at $350 \mathrm{~nm}$ is related to $\pi \rightarrow \pi^{*}$ transitions of the thiocarbonyl group [32]. The wide range of transparency of absorption spectrum of thiourea salt in the visible range as well as the lower value of cut-off wavelength indicates the possibility of using thiourea salt in many applications in the field of higher harmonic generation and optoelectronic applications [33]. On doping with thiourea, UV absorption spectrum of pure PVA has been altered, as represented in Fig. $4 a$. 


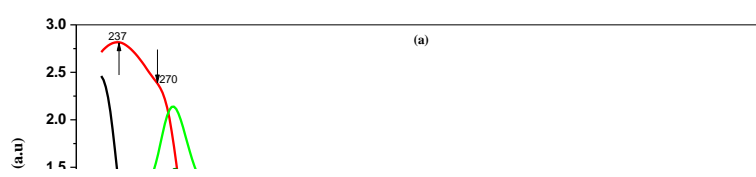

Fig. 4a: The absorbance versus the wavelength for all samples.

The absorption coefficient $(\alpha)$ is calculated using the formula, $\alpha=2.303 \frac{A}{d}$, where, $A$ is the absorbance and $d$ is sample thickness, respectively. Fig. $4 \mathrm{~b}$ shows the relation between the absorbance coefficient and photon energy for all samples. The values of absorption edge are determined for the samples by extrapolating the linear portion of $\alpha$ against ho, as shown in Fig. 4b, to zero absorption value, and listed in Table 1.

The absorption spectra measurement is considered the simplest way to investigate the band structure of the materials. During the absorption process the electrons are stimulated from a state of lower energy to state of higher energy by absorbing a photon of energy known in the transmitting radiation. Hence, changes in transmitted radiation can identify the possible types of electronic transitions. Absorption coefficient $(\alpha)$ for amorphous and semiconductors can be expressed as follows [34]

$$
\alpha=B \frac{\left(h v-E_{g}\right)^{n}}{h v}
$$

Where, $B, h u, E_{g}$ and $n$ are constant, photon energy, optical

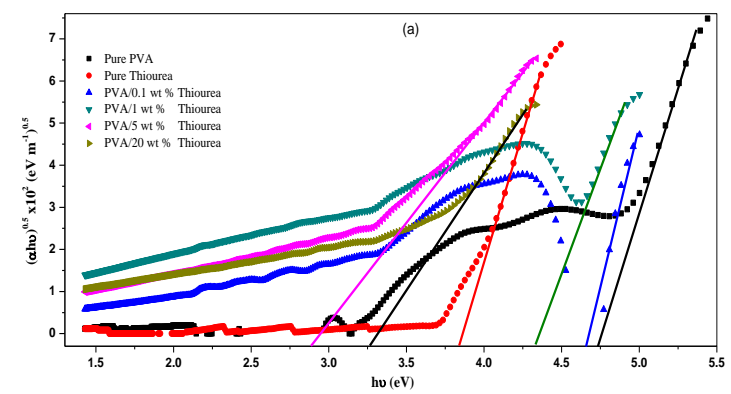

Fig. 5a: $(\alpha h v)^{0.5}$ versus $(h v)$ of all samples.

In the range of low photon energy and near the fundamental absorption edge, it is assumed that, the absorption coefficient $(\alpha)$ is dependent exponentially on $h v$ and follows the empirical Urbach rule as follows [36]:

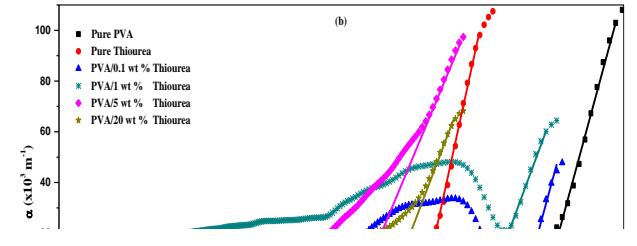

Fig. 4b: The absorbance coefficient versus the photon energy for all samples.

band gap energy and an exponent, respectively. The exponent $n$ depends mainly on the transition nature and is relevant to density of states distribution. For direct allowed transitions, $n$ equals to $1 / 2$ or $3 / 2$, whereas, for indirect forbidden transition $n$ equals to 2 or 3 . It is reported previously, near the edge of fundamental band the indirect and direct transitions is obtained and observed by plotting $(\alpha h v)^{1 / 2}$ and $(\alpha h v)^{2}$ against photon energy $(h v)$ [35]. Fig. 5a\&b displays the variation of $(\alpha h v)^{1 / 2}$ and $(\alpha h v)^{2}$ versus $(h v)$ to estimate indirect and direct optical gap energy values which are summarized in Table 1. It is found that, values of absorption edge, direct and indirect optical gap energy are decreased with doping thiourea salt until a concentration of $5 \mathrm{wt} \%$. For further increasing in doping concentration, these values begin to increase again, as displayed in Fig. 6a. This behavior revealed that, position of the electronic band gap of the doped samples depends mainly on the doping concentration of thiourea. When the dopant concentration is increased, it led to an aggregation of the dopant in the polymer matrix. These aggregations resist the motion of the charge carriers resulting in higher value of optical band gap energy.

Fig. 5b: $(\alpha h v)^{2}$ versus $(h v)$ of all samples.

$$
\alpha=\alpha_{0} \exp \left(\frac{h v}{E_{u}}\right)
$$

Where, $\alpha_{0}$ is a constant and $E_{u}$ is the Urbach energy and denotes to the tail width of localized states in the band gap 
which are associated to disorder and structural defects in the polymer matrix. Fig. $6 \mathrm{~b}$ displays variation of $\ln \alpha$ against $h v$ of pure and doped PVA samples and a linear fit is carried out in the linear parts of the curves to estimate Urbach energy, $\left(E_{u}\right)$ values. Urbach energy values are estimated and summarized in Table 1. It is found that, the values of $E_{u}$ are increased with increasing the doping concentration until

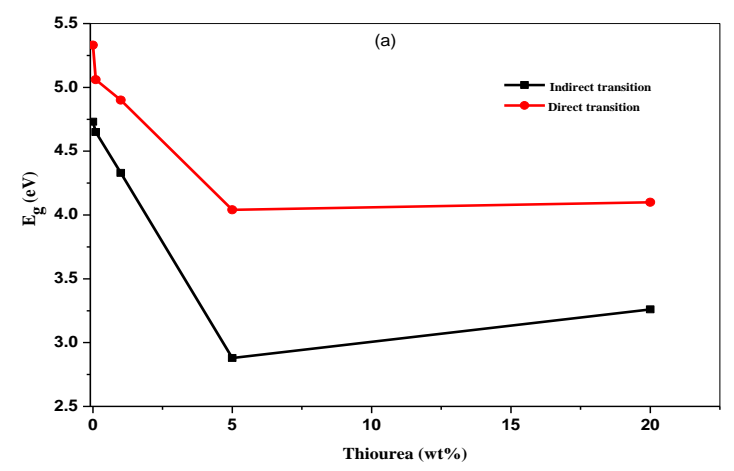

Fig. 6a: Values of optical energy gap versus thiourea wt $\%$.

Table 1: The values of absorbance edge, indirect and direct energy gap, Urbach energy, single oscillator energy $\left(E_{o}\right)$ and dispersion energy $\left(E_{d}\right)$ of all samples. certain concentration and then start to decrease again. The increase in the values of $E_{u}$ suggested that the atomic structural disorder of PVA is affected by thiourea doping. This increase in $E_{u}$ values leads to a redistribution of states, i.e., from band to tail, allowing a great number of possible transitions from the band to the tail and tail to tail [37].

\begin{tabular}{|c|c|c|c|c|c|c|}
\hline Sample & $\begin{array}{l}\text { Absorbance } \\
\text { edge }(\mathrm{eV})\end{array}$ & $\begin{array}{l}\text { indirect energy } \\
\text { gap, }(\mathrm{eV})\end{array}$ & $\begin{array}{l}\text { direct energy } \\
\text { gap, }(\mathrm{eV})\end{array}$ & $\begin{array}{l}\text { Urbach energy } \\
(\mathrm{eV})\end{array}$ & $\begin{array}{l}\text { Single oscillator } \\
\text { energy }\left(E_{o}\right)\end{array}$ & $\begin{array}{l}\text { Dispersion } \\
\text { energy }\left(E_{d}\right)\end{array}$ \\
\hline Pure PVA & 4.81 & 4.73 & 5.33 & 0.35 & 13.67 & 75.95 \\
\hline Pure Thiourea & 3.93 & 3.84 & 4.37 & 0.14 & 4.27 & 1.94 \\
\hline 0.1 wt $\%$ Thiourea & 4.65 & 4.65 & 5.06 & 0.59 & 3.77 & 6.62 \\
\hline $1 \mathrm{wt} \%$ Thiourea & 4.36 & 4.33 & 4.90 & 1.53 & 4.31 & 33.14 \\
\hline 5 wt $\%$ Thiourea & 3.38 & 2.88 & 4.04 & 0.87 & 7.95 & 41.88 \\
\hline 20 wt $\%$ Thiourea & 3.62 & 3.26 & 4.10 & 0.67 & 7.25 & 34.50 \\
\hline
\end{tabular}

\section{Refractive index dispersion}

The refractive index (n) can be estimated using the following formula [38], $n=\frac{1+\sqrt{R}}{1-\sqrt{R}}$, where $\mathrm{R}$ is the reflectance and equals to, $R=1-\sqrt{T^{*} \exp (A)}$ [36], where $\mathrm{T}$ and $\mathrm{A}$ are the transmittance and absorption, respectively. The variation of the refractive index for pure and doped PVA samples versus the wavelength is shown in Fig. 7a. 
Fig. 7a: Refractive index versus the wavelength of all samples.

Fig. 7b: $\left(n^{2}-1\right)^{-1}$ versus $(h v)^{2}$ of all samples.

The dispersion has an effective role in detecting optical materials, because it is an important parameter in design of spectroscopic and optical communication devices. The dispersion of refractive index is attributed to the photon energy $E(v)$ according to the single-oscillator model which explains dielectric response of transitions under the interband absorption edge, as follows [39]

$$
n^{2}-1=\frac{E_{o} E_{d}}{E_{o}-(h v)^{2}}
$$

Where $E_{o}$ and $E_{d}$ are defined as the average excitation energy of the electronic transitions and the dispersion energy, respectively. $E_{o}$ is associated with the optical band gap and $E_{d}$ is considered as a measure of average strength of the interband optical transitions. $E_{d}$ is attributed to the distribution of the charges in the unit cell and depends on the chemical bonding [40]. Fig. 7b shows the variation of $\left(n^{2}-1\right)^{-1}$ against $(h v)^{2}$ for pure and doped samples. Values of $E_{o}$ and $E_{d}$ are calculated by knowing the intercept and slope of the Fig. 7b, and listed in Table 1.

The values of $E_{d}$ and $E_{o}$ are used to determine both static refractive index, $n_{o}$, and static dielectric constant, $\varepsilon_{\mathrm{s}}$, based on the following equation [38]

$$
\begin{aligned}
& n_{o}^{2}=1+\frac{E_{d}}{E_{o}} \\
& \varepsilon_{s}=n_{o}^{2}
\end{aligned}
$$

The relation between refractive index (n) and lattice dielectric constant $\left(\varepsilon_{\mathrm{L}}\right)$ can be expressed as follow [39]

$$
n^{2}=\varepsilon_{L}-\frac{e^{2}}{\pi c^{2}}\left(\frac{Y}{m^{*}}\right) \lambda^{2}
$$

Where, $e, c,\left(Y / m^{*}\right)$ and $\lambda$ are the electronic charge, speed of light, ratio of carrier concentration to effective mass and wavelength, respectively. Fig. 8 shows the variation of $n^{2}$ versus $\lambda^{2}$. The values of $\left(Y / m^{*}\right)$ and $\left(\varepsilon_{\mathrm{L}}\right)$ are calculated by knowing slope and the intercept of Fig. 8 and summarized in Table 2. The difference between static and lattice dielectric constants values may be due to the contribution of free carriers in the different samples.

Fig. 8: The variation of $n^{2}$ versus $\lambda^{2}$ for all samples. 
It is already well known that, the dielectric constant in the range of transparency can be written as follows;

$$
n^{2}=\varepsilon_{L}-\frac{\omega_{p}^{2}}{\omega^{2}}
$$

and

$$
\omega_{p}^{2}=\frac{e^{2} \cdot\left(Y / m^{*}\right)}{\varepsilon_{0}}
$$

Where, $\omega_{\mathrm{p}}, \omega$, e and $\varepsilon_{0}$ are defined as plasma frequency, frequency of the incident light, electron charge and permittivity of free space $\left(8.854 \times 10^{-12} \mathrm{~F} / \mathrm{m}\right)$, respectively. The values of plasma frequency of the investigated samples are estimated and listed in Table. 2.

\begin{tabular}{|c|c|c|c|c|c|}
\hline Sample & $\begin{array}{l}\text { Static refractive } \\
\text { index, } n_{o}\end{array}$ & $\begin{array}{l}\text { Static dielectric } \\
\text { constant, } \varepsilon_{S}\end{array}$ & $\begin{array}{l}\text { Lattice dielectric } \\
\text { constant, } \varepsilon_{L}\end{array}$ & $\begin{array}{c}\mathrm{Y} / \mathrm{m}^{*} \\
\left(10^{41} \mathrm{~m}^{-3} \mathrm{~g}^{-1}\right)\end{array}$ & $\begin{array}{c}\text { Plasma frequency, } \\
\omega_{\mathrm{p}(\mathrm{Hz})}\end{array}$ \\
\hline Pure PVA & 2.56 & 6.56 & 15.96 & 176 & $2.26 \times 10^{8}$ \\
\hline Pure Thiourea & 1.21 & 1.45 & 35.95 & 396 & $3.38 \times 10^{8}$ \\
\hline 0.1 wt $\%$ Thiourea & 1.66 & 2.76 & 3.87 & 1.71 & $2.22 \times 10^{7}$ \\
\hline $1 \mathrm{wt} \%$ Thiourea & 2.95 & 8.69 & 7.42 & 0.95 & $1.65 \times 10^{7}$ \\
\hline 5 wt $\%$ Thiourea & 2.50 & 6.27 & 6.90 & 3.06 & $2.97 \times 10^{7}$ \\
\hline $20 \mathrm{wt} \%$ Thiourea & 2.40 & 5.76 & 6.36 & 1.70 & $2.21 \times 10^{7}$ \\
\hline
\end{tabular}

Table 2: Values of static refractive index, $n_{o}$, static dielectric constant, $\varepsilon_{s}$, lattice dielectric constant, $\varepsilon_{L}$ and Y/m* of all samples.

\section{CONCLUSION}

Structure and optical properties of thiourea-PVA composites have been examined using different techniques such as, XRD, FTIR, Raman spectroscopy and UV-Vis spectroscopy. XRD results showed that thiourea is highly crystalline material with an intense sharp peak at $2 \theta=20.98^{\circ}$ and the crystallinity of thiourea doped PVA samples increased with increasing the thiourea content. FTIR and Raman spectroscopy are used to investigate the change in the molecular structure. The analysis of UV-Vis data revealed that, values of absorption edge, direct and indirect optical gap energy are decreased with doping thiourea salt until a concentration of $5 \mathrm{wt} \%$ and then start to increase again. Moreover, values of dispersion energy $\left(E_{d}\right)$ and single oscillator energy $\left(E_{o}\right)$ have been changed with the thiourea concentration as observed for direct and indirect optical energy. This behavior has been interpreted based on the aggregation of the thiourea dopant in the PVA polymer matrix. Static and lattice dielectric constants have been estimated.

\section{ACKNOWLEDGMENT}

One of authors (T. Fahmy) would like to thank Prince Sattam bin Abdulaziz University, Scientific Research Deanship, KSA. Also, one of the authors (ZME) would like to thank Deanship of Scientific Research in Princes Nourah bint Abdulrahman University, Saudi Arabia for funding this research through the fast-track research funding program.

\section{REFERENCES}

[1] Deb S and Sarkar D 2018 "Effect of annealing temperature on optical properties of silver-PVA nanocomposite", Optik 157, 1115.

[2] Tripathia J, Keller J M, Dasc K, Tripathi S, Fatima A and Shripathi T 2012 "Structural, optical and chemical characterization of Rhodamine (B) doped poly (vinyl) alcohol films" Applied Surface Science, 261, 481.

[3] Patil D S, Shaikh J S, Dalavi D S, Kalagi S S and Patil P S 2011 "Chemical synthesis of highly stable PVA/PANI films for supercapacitor application”, Materials Chemistry and Physics, 128, 449.

[4] Fahmy T 2001 "Dielectric Relaxation and Electrical Conductivity Study in Thiourea- Doped Poly (Vinyl Alcohol)", Inter J. Polym. Mater. 50, 109.

[5] Siddaiaha T, Ojhaa P, Kumara N O and Ramu C 2018 "Structural, Optical and Thermal Characterizations of PVA/MAA:EA Polyblend Films", Materials Research 21(5), e20170987.

[6] Kim K H and Jo W H 2008 "Improvement of tensile properties of poly(methyl methacrylate) by dispersing multi-walled carbon nanotubes functionalized with poly(3-hexylthiophene)-graft-poly(methyl methacrylate)", Composites Sci. \& Tech. 68, 2120.

[7] Madhurambal G and Mariappan M 2010 "Growth and characterization of urea-thiourea nonlinear optical organic mixed crystal", Indian J. Pure \& Appl. Phys. 48, 264. 
[8] Chemla S and Zyss J 1987 "Nonlinear Optical Properties of Organic Molecules and Crystals”, Vol. 1, Academic Press, NY.

[9] Prasad P N and Williams D J, 1991 “Introduction to Nonlinear Optical Effects in Organic Molecules and Polymers", Wiley, NY.

[10] Fahmy T and Ahmed M T 2015 "Electrical Transport and Magnetoresistance of Doped Polypyrrole", Inter. J. Eng.\&Appl. Sci.,2, 56.

[11] Omkarama I, Chakradharb R P and Rao J L 2007 "EPR, optical, infrared and Raman studies of $\mathrm{VO}^{2+}$ ions in polyvinylalcohol films", Physica B, 388, 318-325.

[12] Migahed M D, Fahmy T, Ishra M and Barakat A 2004 "Preparation, characterization, and electrical conductivity of polypyrrole composite films", Polymer Testing, 23, 361.

[13] Fahmy T and Ahmed M T 2003 “Alternating -Current Conductivity and Dielectric Relaxation of Poly (Acrylonitrile-Butadiene-Styrene) Terpolymer Doped with Tetrabutylammonium Tetrafluoroborate", J. Polym. Mater. 20, 367.

[14] Migahed M D and Fahmy T 1994 "Structural relaxation around the glass-transition temperature in amorphous polymer blends: temperature and composition dependence", Polymer 35, 1688.

[15] Fahmy T and Ahmed M T 2001 "Thermal induced structural change investigations in PVC/PEMA polymer blend", Polymer Testing 20, 477.

[16] Ahmed M T and Fahmy T 2000 "Carrier Transport and Thermally Stimulated Depolarization Current in Iodine-Doped Poly (Vinyl Chloride)", J. Polym. Mat. $17,133$.

[17] Deshmukh S H, Burghate D K, Shilaska S N and Deshmukh P T 2008 "Optical properties of polyaniline doped PVC-PMMA thin films”, Indian J. Pure \& Appl. Phys. 46, 344.

[18] Fahmy T, Sarhan A, Elsayed I A and Abelwahed H G 2018 "Optical Properties of Poly (Vinyl Chloride-coVinyl Acetate-co-2-Hydroxypropyl Acrylate)/ (Acrylonitrile-Butadiene-Styrene) Blends", Inter. J. of Eng. Research \&Tech., 11(9), 1405.

[19] Fahmy T, Sarhan A, Elsayed I A and Ahmed M T 2018 "Effect of UV Irradiation on The Structure and Optical Properties of PVA/Cucl 2 ", J. of Advances in Phys., 14(2) 5378.

[20] Jayalakshmi D and Kumar J 2006 Cryst. Res. Technol. 41(1) 37.

[21] Sheela T, Bhajantri R F, Ravindrachary V, Rathod S G, Pujari P K, Poojary B and Somashekar R 2014 "Effect of UV irradiation on optical, mechanical and microstructural properties of PVA/NaAlg blends", Radiation Phys. \& Chem. 10345.
[22] Zhang X, Zhang J and Liu Z 2005 "Tubular composite of doped polyaniline with multi-walled carbon nanotubes", Appl. Phys. A 801813.

[23] Abdel-Fattah E, Alharthi A I and Fahmy T 2019 Applied Physics A 125475.

[24] Rozenberg M 1983 Polymers Based on Vinyl Acetate, Khimiya, Leningrad.

[25] Alia J M, Edwards H G M and Moore J $995 \mathrm{~J}$. of Raman Spectroscopy 26715.

[26] Ragamathunnisa M, Jasmine V R E, Padmavathy R and Radha N 2013 IOSR J. of Appl. Phys., 4(1) 5.

[27] Nakamoto K 1978 Infrared and Raman spectra of inorganic and coordination compounds, ch. 5-8, $5^{\text {th }}$ Ed., Wiley, London.

[28] Cao P, Yao J, Ren B, Gu R and Tian Z 2002 J. Phys. Chem. B 10610150.

[29] Thomas S and Stuart B H 1997 Spectrochim. Acta Part A 532275.

[30] Kuptsov A. Kh and Zhizhin G N 1998 Handbook of Fourier Transform Raman and Infrared Spectra of Polymers, Elsevier, Amsterdam, The Netherlands.

[31] Moroso D, Cella A and Peccatori E 1987 Chim. Ind. (Milan) 251897.

[32] Rao C N R 1975 Ultra-Violet and Visible Spectroscopy $3^{\text {rd }}$ Ed., Butterworth \& Co Publishers, Ltd, p. 29.

[33] Madhavan J, Aruna S, Anuradha A, Premanand D, Potheher I V, Thamizharasan K and Sagayaraj P 2007 Opt. Mater. 291211.

[34] Tauc J, Menth A and Wood D 1970 Phys. Rev. Lett. 25 749.

[35] Davis P W and Shilliday T S 1960 Phys. Rev., 118(4) 1020.

[36] Urbach F 1953 Phys. Rev. 921324.

[37] Yahia I S, Farag A A M, Cavas M and Yakuphanoglu F 2013 Superlattices and Microstructures 53(1) 63.

[38] Pankove J I 1971 Optical Processes in Semiconductors", Prentice Hall, NY, USA.

[39] Wemple S H and DiDomenico Jr. M 1971 Phys. Rev. B 3(4) 1338.

[40] Afak H S, Merdan M and Yüksel O F 2002 Turkish J. Phys. 26(5) 341. 